\title{
Chandra X-ray Observations of the Quadruply Lensed Quasar RX J0911.4+0551
}

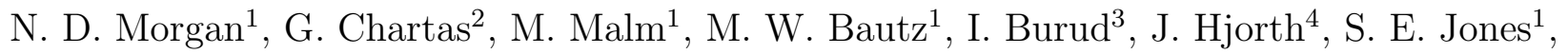 \\ P. L. Schechter ${ }^{1}$
}

\begin{abstract}
We present results from X-ray observations of the quadruply lensed quasar RX J0911.4+0551 using data obtained with the Advanced CCD Imaging Spectrometer (ACIS) on board the Chandra X-ray Observatory. The 29 ks observation detects a total of $\sim 404$ X-ray photons $(0.3$ to $7.0 \mathrm{keV})$ from the four images of the lensed quasar. Deconvolution of the aspect corrected data resolves all four lensed images, with relative positions in good agreement with optical measurements. When compared to contemporaneous optical data, one of the lensed images (component A3) is dimmer by a factor of $\sim 6$ in X-rays with respect to the 2 brighter images (components A1 and A2). Spectral fitting for the combined images shows significant intrinsic absorption in the soft (0.2 to $2.4 \mathrm{keV})$ energy band, consistent with the mini-BAL nature of this quasar, while a comparison with ROSAT PSPC observations from 1990 shows a drop of $\sim 6.5$ in the total soft bandpass flux. The observations also detect $\sim 157 \mathrm{X}$-ray photons arising from extended emission of the nearby cluster (peaked $\sim 42^{\prime \prime}$ SW of RX J0911.4+0551) responsible for the large external shear present in the system. The Chandra observation reveals the cluster emission to be complex and non-spherical, and yields a cluster temperature of $k T=2.3_{-0.8}^{+1.8} \mathrm{keV}$ and a 2.0 to $10 \mathrm{keV}$ cluster luminosity within a $1 \mathrm{Mpc}$ radius of $L_{X}=7.6_{-0.2}^{+0.6} \times 10^{43} \mathrm{ergs} \mathrm{s}^{-1}$ (error bars denote $90 \%$ confidence limits). Our mass estimate of the cluster within its virial radius is
\end{abstract}

\footnotetext{
${ }^{1}$ Department of Physics, Massachusetts Institute of Technology, Cambridge MA 02139; malm@space.mit.edu, mwb@space.mit.edu, ndmorgan@space.mit.edu, schech@space.mit.edu, sjones@space.mit.edu

${ }^{2}$ Department of Astronomy and Astrophysics, 525 Davey Laboratory, Pennsylvania State University, University Park, PA 16802; chartas@lonestar.astro.psu.edu

${ }^{3}$ Institut d'Astrophysique et de Geophysique de Liège, Universié de Liège, Avenue de Cointe 5, 4000 Liège, Belgium; burud@astro.ulg.ac.be

${ }^{4}$ Astronomical Observatory, University of Copenhagen, Juliane Maries Vej 30, DK-2100 Copenhagen, Denmark; jens@astro.ku.dk
} 
$2.3_{-0.7}^{+1.8} \times 10^{14} \mathrm{M}_{\odot}$, and is a factor of two smaller than, although consistent with, previous mass estimates based on the observed cluster velocity dispersion.

Subject headings: gravitational lensing: individual (RX J0911.4+0551) - X-rays: general - quasars: individual (RX J0911.4+0551) — quasars: (BAL)

\section{Introduction}

Gravitational lenses that produce multiple images of quasars can be used to measure cosmological parameters (Refsdal 1964; Kochanek 1996), to study the properties of the lensing galaxies (Keeton, Kochanek, \& Falco 1998) and to resolve structures associated with the lensed quasar (Bunker, Moustakas, \& Davis 2000). For the past twenty years such work has been carried out at optical and radio wavelengths. With the advent of the subarcsecond angular resolution of the Chandra X-ray Observatory (Weisskopf, O’Dell, \& van SpeyBroeck 1996), one can hope to carry out similar studies at X-ray wavelengths. As the X-ray emission from quasars has different spatial and temporal structure, X-ray studies offer a variety of new opportunities. In the present paper we report on Chandra observations of the quadruply imaged quasar RX J0911.4+0551, which have implications both for the structure of the lensing potential and for the structure of the X-ray emitting region.

To our knowledge, the observations of RX J0911.4+0551 presented here are the first X-ray observations of the system since its detection by the ROSAT All Sky Survey in 1990 October. The source was optically identified as a gravitationally lensed $z=2.8$ quasar by Bade et al. (1997), and later confirmed as a quadruple system by Burud et al. (1998). Lensing models require a large external shear $(\gamma \gtrsim 0.15)$ to account for the system's complex image geometry. Burud et al. (1998) first suggested the source of this shear to be a group of galaxies in the southwest vicinity of RX J0911.4+0551, which has been spectroscopically confirmed by Kneib et al. (2000) to be a high-redshift cluster of at least 24 galaxies at $\bar{z}=0.769$. The original ROSAT observation of RX J0911.4+0551 lacked the sensitivity and angular resolution required for a detailed X-ray analysis of the lensed quasar and cluster. Deconvolution of the Chandra observations presented here identifies all four components of the lensed quasar, and an adaptively smoothed image reveals the extent of the X-ray emission from the nearby cluster as well. The format of the paper is as follows: we describe the Chandra observations and reductions of RX J0911.4+0551 in §2, discuss X-ray astrometry and relative photometry of the lens components in $\$ 2.1$, and report spectral properties of the lensed quasar in $\S 2.2$. The X-ray detection and analysis of the cluster is presented in $\S 3$. We summarize our findings and briefly comment on possible sources of X-ray variability for the system in $\S 4$. 


\section{Observations and Analysis}

RX J0911.4+0551 was observed for $\sim 29 \mathrm{ks}$ with the Chandra X-ray Observatory on 1999 November 2. The data were obtained using the back-illuminated S3 chip of the Advanced CCD Imaging Spectrometer (ACIS; Garmire et al. 1992; Bautz et al. 1998) at a focal plane temperature of $-110^{\circ} \mathrm{C}$. The telescope pointing placed RX J0911.4+0551 at the default aim point for the ACIS-S array during the exposure. Three periods of background flaring were detected, during which time the count rate reached 2 to 4 times the nominal background rate. Since the contamination of point sources from background flaring is small, these times were not filtered from our observation except for the cluster analysis discussed in $\S 3$. Removal of the three X-ray flares reduces the effective exposure time of the data to $\sim 26 \mathrm{ks}$.

The data were reduced using the CIAO software tools, following the science threads outlined on the Chandra X-ray Observatory Center (CXC) user support homepage. The data used throughout this paper has been reprocessed by the CXC using software version R4CU5UPD9, which results in an improved aspect solution over earlier versions of the processing software. Also, in the standard CXC pipeline, the photon event positions are randomized by \pm 0 '.246 (1 ACIS pixel $=0$ '!492), which is mainly to reduce aliasing effects for observations of duration less than $\sim 2 \mathrm{ks}$. Given the small angular extent of the lens $\left(\sim 3^{\prime \prime}\right)$, we reproduced the event positions using the CXC tool acis_process_events without incorporating the randomization.

Throughout this work, we have adopted a flat cosmological model parameterized by $q_{o}=0.5$ and $H_{o}=50 \mathrm{~km} \mathrm{~s}^{-1} \mathrm{Mpc}^{-1}$. At the redshift of the lensing galaxy $(z=0.769$; Kneib

et al. 2000), this gives a physical scale of $8.2 \mathrm{kpc} \operatorname{arcsec}^{-1}$. Unless otherwise noted, error bars denote $90 \%$ confidence limits.

\subsection{Relative Astrometry and Photometry}

In Figure 1a we show the X-ray image of RX J0911.4+0551, resampled at a resolution of $0^{\prime \prime} .1$ pixel $^{-1}$ and smoothed with a Gaussian of $\sigma=0^{\prime \prime} .2$. A total of $\sim 404 \mathrm{X}$-ray photons in the 0.3 to $7.0 \mathrm{keV}$ bandpass are estimated from the four lensed images of the quasar, which compensates for contributions from the X-ray background and from the nearby cluster. Adopting the nomenclature of Burud et al. (1998), component B is clearly resolved whereas components A1, A2 and A3 are not clearly separated. To enhance the image quality we applied the Richardson-Lucy maximum likelihood deconvolution technique (Richardson 1972; Lucy 1974) to the X-ray image of RX J0911.4+0551. The point spread function (PSF) incorporated in the deconvolution was generated with the raytrace simulator MARX v3.0 (Wise 
et al. 1997). The input spectrum for the simulation was based on the model of the observed spectrum of RX J0911.4+0551 (see §2.2). In particular, we chose an absorbed power law model with Galactic absorption of $\mathrm{N}_{H}=0.036 \times 10^{22} \mathrm{~cm}^{-2}$, intrinsic absorption at $z=2.8$ of $\mathrm{N}_{H}=4.1 \times 10^{22} \mathrm{~cm}^{-2}$ and a photon spectral index of 1.63 . The PSF and image were then sampled at a resolution of $00^{\prime \prime} 1$. In Figure $1 \mathrm{~b}$ we show the deconvolved image of RX J0911.4+0551 smoothed with a Gaussian of $\sigma=0$ '.2. All four components are now resolved with relative positions in good agreement (to better than 0 '.15) with those found from optical observations. Component B appears somewhat extended along the EW direction in both the raw and deconvoled images, which we attribute to the small number of counts $(\sim$ 70 in total for 0.3 to $7.0 \mathrm{keV}$ ) detected for this component. In Table 1 we present the optical and X-ray offsets of the lensed images.

The ACIS PSF (when properly normalized) gives the probability distribution of detecting a single X-ray photon at some position with respect to the center of a point source. One can therefore use several PSFs, of different relative intensities, to quantify the likelihood of obtaining the observed distribution of photon counts for a given set of image flux ratios and positions (e.g., see Cash 1979). Specifically, we have solved for the relative flux ratios of the system components using the Cash (1979) $C$ statistic to quantify the relative goodness of fit between the observed and predicted number of photons per bin. The system was modeled using four of the MARX PSFs described above; relative positions of the four sources were held fixed according to the optical astrometry listed in Table 1, but the overall position of the system was allowed to vary during the fit. The best fit parameters were then determined by simultaneously varying the intensity of each component and the overall position of the system using Powell's direction-set method (Press et al. 1995) until a minimum $C$ statistic was reached. This analysis was performed on the raw data at a resampling size of 0 '.06, or an eighth of an ACIS pixel.

In Table 2 we present our optimal X-ray flux ratios for the 0.3 to $7.0 \mathrm{keV}$ bandpass, along with contemporaneous (to within one day) $I$-band data obtained from an ongoing monitoring campaign of RX J0911.4+0551 using the Nordic Optical Telescope (NOT; Hjorth et al. 2001). Since the separation between components A1 and A2 is 0.48, or slightly less than one ACIS pixel, it is prudent to discuss the combined flux of components A1 and A2 when comparing to the optical data. Our maximum likelihood analysis yields an (A1 + A2)/B X-ray flux ratio of 4.68 and an $(\mathrm{A} 1+\mathrm{A} 2+\mathrm{A} 3) / \mathrm{B}$ flux ratio of 4.87 , which are $\sim 20 \%$ and $\sim 30 \%$ smaller than the respective optical ratios of 5.67 and 6.95. A significant difference is observed for $\mathrm{A} 3 / \mathrm{B}$, where we find an X-ray flux ratio of 0.19 as compared to the optical ratio of 1.28. If we normalize the optical and X-ray fluxes at their respective $(\mathrm{A} 1+\mathrm{A} 2)$ values, then component $\mathrm{B}$ is brighter by a factor of 1.2 in X-rays and component A3 is fainter by a factor of 5.7 in X-rays. We briefly discuss possible implications of the faintness of A3 in $\S 4$. 


\subsection{Spectral Properties}

A source spectrum of RX J0911.4+0551 was extracted by combining events from all four quasar images and a background spectrum from events located within an annulus centered on RX J0911.4+0551 with inner and outer radii of $15^{\prime \prime}$ and $30^{\prime \prime}$, respectively. Redistribution matrices and ancillary response files were generated using the CXC tools mkrmf and mkarf. A fit of an absorbed power-law model with intrinsic and Galactic absorption in the energy range 0.5-6.0 keV yields a photon spectral index of $1.45_{-0.20}^{+0.24}$ and a $0.2-2.4 \mathrm{keV}$ flux (approximating the ROSAT bandpass) of $\mathrm{f}_{X}=3.3_{-0.1}^{+0.1} \times 10^{-14} \mathrm{erg} \mathrm{s}^{-1} \mathrm{~cm}^{-2}$. A fit of a simple absorbed powerlaw model with Galactic absorption of $\mathrm{N}_{H}=0.036 \times 10^{22} \mathrm{~cm}^{-2}$, considering only energies above $1.5 \mathrm{keV}$, yields a photon spectral index of $2.22_{-0.48}^{+0.43}$, which is typical for high redshift radio-quiet quasars (Reimers et al. 1995). As we show in Figure 2, extrapolating this model to energies below $1.5 \mathrm{keV}$ clearly indicates that considerable intrinsic absorption is present. This low-energy absorption is consistent with the mini-BAL nature of RX J0911.4+0551 (Bade et al. 1997), and has been observed in at least one other BAL quasar (e.g., PHL 5200, Mathur et al. 1995). For RX J0911.4+0551, the 0.2-2.4 keV flux of the unabsorbed model is $\mathrm{f}_{X}=1.0_{-0.4}^{+0.5} \times 10^{-13} \mathrm{erg} \mathrm{s}^{-1} \mathrm{~cm}^{-2}$, and represents what we would expect to observe were the absorber not present.

RX J0911.4+0551 was observed with the ROSAT PSPC on 1990 October with a countrate of $0.020 \pm 0.0088 \mathrm{cnts} \mathrm{s}^{-1}(1 \sigma)$. Assuming the simple absorbed power-law model from above, the corresponding X-ray flux for the $R O S A T$ observation is $\mathrm{f}_{X}=(2.2 \pm 1.1)$ $\times 10^{-13} \mathrm{erg} \mathrm{s}^{-1} \mathrm{~cm}^{-2}(1 \sigma)$. This includes emission from both the gravitational lens and extended emission from the nearby cluster (see §3). Within a circular aperture centered on RX J0911.4+0551 of diameter $1^{\prime}$ (twice the half-power diameter of the ROSAT PSPC), the Chandra estimate of the flux due to solely cluster emission is $(6.6 \pm 1.0) \times 10^{-15} \mathrm{ergs}^{-2}$ $\mathrm{s}^{-1}(1 \sigma)$. Therefore, the cluster contribution to the original ROSAT flux was small, on the order of a few percent. When we compare the estimated ROSAT flux from RX J0911.4+0551 to the Chandra observation, we find it has dropped by a factor of $\sim 6.5 \pm 3.3(1 \sigma)$ over the 10-year period. Our spectral fits to the Chandra spectrum of RX J0911.4+0551 indicate that a factor of $3.0 \pm 1.5(1 \sigma)$ variation in the $0.2-2.4 \mathrm{keV}$ flux is possible if one assumes that the presently observed intrinsic X-ray absorber were not present during the ROSAT PSPC observation.

\section{Cluster Analysis}

The Chandra observation of RX J0911.4+0551 provides the first detailed X-ray study of the nearby cluster reported by Kneib et al. (2000). The extent of the X-ray emission from 
the cluster is apparent after adaptively smoothing the image with the CXC tool csmooth (Ebeling, White and Rangarajan, 2000). Contours for the smoothed image are displayed in Figure 3, and are shown overlaid on an IJK composite exposure of the RX J0911.4+0551 field from Burud et al. (1998). When creating the smoothed image, we have used a conservative lower bound on the signal to noise ratio $(\mathrm{SN})$ of the smoothing kernel of $\mathrm{SN} \geqslant 3$, and have also excluded emission from within a small ( $\sim 4^{\prime \prime}$ radius) region around RX J0911.4+0551 itself. The smoothed emission profile consists of a bright, extended component peaked $\sim 42^{\prime \prime}$ southwest of component B, but is clearly elongated in the direction toward RX J0911.4+0551. An iterative centroiding algorithm (Buote \& Canizares 1992) applied to the binned event positions yields a centroid for the extended emission of $\Delta \alpha=-12^{\prime \prime} \cdot 0, \Delta \delta=-39$ !'8, where offsets are with respect to the centroid of component B. The contour morphology suggests that the cluster mass distribution is complex and non-spherical, and possibly not dynamically relaxed. If the X-ray emission follows the mass distribution (which, simulations suggest, it need not always do during merging; see, e.g. Roettiger, Stone \& Mushotzky 1998) then our image implies that the cluster is responsible for some fraction of the mass convergence at the location of the lens.

Within an ellipse of $70^{\prime \prime} \times 90^{\prime \prime}$ (dashed outline in Figure 3) encompassing the bulk of the cluster emission, we find a total of 157 net cluster counts above an estimated background of 222 counts. The corresponding 0.3 to $7.0 \mathrm{keV}$ flux (assuming the spectral form described below $)$ is $\mathrm{f}_{X}=(2.5 \pm 0.2) \times 10^{-14} \mathrm{erg} \mathrm{s}^{-1} \mathrm{~cm}^{-2}(1 \sigma)$.

To estimate an X-ray temperature for the cluster, we extracted a 0.3 to $7.0 \mathrm{keV}$ source spectrum centered on the SW peak within a 20" aperture, and subtracted a background spectrum from an annulus with inner and outer radii of 90" and 140", respectively. Fitting the emission spectrum to an optically thin Raymond and Smith (1977) plasma model with a Galactic absorption of $N_{H}=0.036 \times 10^{22} \mathrm{~cm}^{-2}$, a metal abundance of $0.3 \mathrm{Z}_{\odot}$, and a cluster redshift of $z=0.769$ (Kneib et al. 2000) yields a temperature of $k T=2.3_{-0.8}^{+1.8}$ at the $90 \%$ confidence level. This is consistent with the estimate of $k T=4.5 \pm 1.2 \mathrm{keV}$ obtained by Kneib et al. (2000) from their measurement of the cluster velocity dispersion and the $\sigma-T_{x}$ relationship (Girardi et al. 1996).

We do not detect enough photons to constrain the radial surface brightness profile of the cluster. We obtain a crude estimate of the integrated cluster emission by assuming the profile is well-described by an isothermal $\beta$-model (Cavaliere \& Fusco-Femiano 1976) with parameters typical for nearby, non-cooling flow clusters $\left(\beta=0.6, r_{c}=0.22 \mathrm{Mpc}\right.$; Mohr et al. 1999). Normalizing the model using the observed flux within the elliptical aperture, we predict a 2.0 to $10 \mathrm{keV}$ luminosity within a $1 \mathrm{Mpc}\left(122^{\prime \prime}\right)$ radius of $L_{X}=7.6_{-0.2}^{+0.6} \times 10^{43}$ ergs $\mathrm{s}^{-1}$. 
From our temperature we may obtain a crude cluster mass estimate. We first estimate the virial radius for the cluster. Girardi et al. (1998) find, for nearby clusters, that the

virial radius is $R_{V} \approx 4 \sigma_{1000} h_{50}^{-1} \mathrm{Mpc}$, where $\sigma_{1000}$ is the radial velocity dispersion divided by $1000 \mathrm{~km} \mathrm{~s}^{-1}$. Assuming the virial radius scales with redshift as $R_{V} \sim(1+z)^{-3 / 2}$, and given the observed velocity dispersion $\sigma_{1000}=0.84$ (Kneib et al. 2000), we estimate $R_{V} \approx 1.4 h_{50}^{-1} \mathrm{Mpc}$. Assuming an isothermal plasma with the density distribution described above and $k T=2.3 \mathrm{keV}$, the mass within the virial radius is $M\left(R_{V}\right)=2.3_{-0.7}^{+1.8} \times 10^{14} \mathrm{M}_{\odot}$. Alternatively, the mass-temperature relation of Hojrth, Oukbir, \& van Kampen (1998) gives $M\left(R_{V}\right)=3.2_{-1.0}^{+2.5} \times 10^{14} \mathrm{M}_{\odot}$. Here the $90 \%$ confidence limits are dominated by errors in the temperature, and do not include systematic errors arising from our ignorance of the slope of the surface brightness distribution, or from calibration uncertainties in the mass-temperature relationship. Our X-ray mass estimate is somewhat smaller than, although consistent with, the virial estimate of $4.8_{-2.0}^{+2.3} \times 10^{14} \mathrm{M}_{\odot}\left(1 \sigma ; \Omega_{m}=1.0, \Omega_{\Lambda}=0.0\right)$ obtained by Kneib et al. (2000). Given the asymmetry of the X-ray emission, it is reasonable to speculate that the X-ray gas is not in hydrostatic equilibrium. In this case, one might well expect the emission weighted X-ray temperature to lead to an underestimate of the gravitating mass (e.g. Roettiger, Stone and Mushotzky 1998). In any event, given the large uncertainties, the agreement among the mass estimates must be regarded as good.

\section{Discussion and Conclusions}

Our analysis has detected two indications of X-ray variability for RX J0911.4+0551. First, component A3 is dimmer in X-rays by a factor of $\sim 6$ when compared to contemporaneous optical data. The reason for A3's X-ray faintness is not known. One possible explanation is differential $N_{H}$ column densities along the lines of sight to the quasar images. Although an attempt was made to search for differential X-ray extinction between the four lens components (the details of which will be presented in a forthcoming paper; Chartas et al. 2001), a preliminary analysis is inconclusive; the intrinsic $N_{H}$ column density of image A3 is poorly constrained by the Chandra data. A demagnifying microlensing event of component A3 is also a possible, although somewhat unlikely, explanation. A six-fold drop in flux would require fluctuations of the order $\Delta m \sim 2$ magnitudes; microlensing dips of this size are rare in theory (Witt, Mao, \& Schechter 1995).

Second, the total observed flux for the system has dropped by a factor $\sim 6.5$ when compared to ROSAT PSPC observations obtained in 1990 October. Such a drop in flux may be a result of quasar engine variability. Almaini et al. (2000) have observed engine variability of a factor of three in flux over a period of several days, so a factor of $\sim 6.5$ 
between the ROSAT and Chandra observations is not unlikely.

In conclusion, the unique imaging capabilities of Chandra has resolved all four images of the quadruply lensed system RX J0911.4+0551 (detecting $\sim 404$ photons in the 0.3 to $7.0 \mathrm{keV}$ energy range), with relative image positions in good agreement with optical measurements. In addition to the two indications of X-ray variability described above, we have also detected strong soft $(<1.5 \mathrm{keV})$ X-ray absorption in the spectra of the combined quasar images, which is consistent with the mini-BAL nature of the quasar. The Chandra observation of RX J0911.4+0551 has also identified extended X-ray emission from the nearby cluster, detecting $\sim 157$ photons $\left(0.3\right.$ to $7.0 \mathrm{keV}$ ) in a region of $\sim 1.4 \operatorname{arcmin}^{2}$. The morphology of the cluster emission suggests a complex and non-spherical cluster mass distribution. Our estimates of the cluster X-ray temperature $\left(k T=2.3_{-0.8}^{+1.8} \mathrm{keV}\right)$ and virial mass $\left(2.3_{-0.7}^{+1.8} \times\right.$ $10^{14} \mathrm{M}_{\odot}$ ) are consistent with previous estimates derived from the observed velocity dispersion of the cluster galaxies (Kneib et al. 2000).

N.D.M. and P.L.S. gratefully acknowledge support from the U.S. National Science Foundation through grant AST96-16866 and from NASA under contract NAS-8-37716. M.W.B. and M.M. acknowledge support from NASA under contracts NAS-8-37716, NAS-8-38252 and 1797-MIT-NA-A-38252. J. H. acknowledges support by the Danish Natural Science Research Council (SNF).

\section{REFERENCES}

Almaini, O., Lawerence, A., Shanks, T., Edge, A., Boyle, B. J., Georgantopoulos, I., Gunn, K. F., Stewart, G. C., \& Griffiths, R. E. 2000, MNRAS, 315, 325

Bade, N., Siebert, J., Lopez, S., Voges, W., \& Reimers, D. 1997, A\&A, 317, L13

Bautz, M. W., et al. 1998, X-Ray Optics, Instruments and Missions, ed. R. B. Hoover \& A. B. Walker, Proc. SPIE, 3444, 210

Bunker, A. J., Moustakas, L. A., \& Davis, M. 2000, ApJ, 531, 95

Buote, D. A., \& Canizares, C. R. 1992, ApJ, 400, 385

Burud, I., Courbin, F., Lidman, C., Jaunsen, A. O., Hjorth, J., Østensen, R., Andersen, M. I., Clasen, J. W., Wucknitz, O., Meylan, G., \& Magain, P. 1998, ApJ, 501, L5

Cash, W. 1979, ApJ, 228, 939 
Cavaliere, A. \& Fusco-Femiano, R. 1976, A\&A, 49, 137

Chartas et al. 2001, in preparation.

Ebeling, H., White, D. A., \& Rangarajan, F. V. N. 2000, submitted to MNRAS.

Garmire, G. P. et al. 1992, AIAA, Space Programs and Technologies Conference, Huntsville, AL, Mar 24-27

Girardi, M., Fadda, D., Giuricin, G., Mardirossian, F., Mezzetti, N., \& Biviano, A. 1996, ApJ, 457, 61

Girardi, M., Borgani, S., Giuricin, G., Mardirossian, F., \& Mezzetti, M. 1998, ApJ, 505, 74

Hjorth, J., Oukbir, J., \& van Kampen, E. 1998, MNRAS, 298, L1

Hjorth et al. 2001, ApJ, in preparation

Keeton, C. R., Kochanek, C. S., Falco, \& E. E. 1998, ApJ, 509, 561

Kneib, J.-P., Cohen, J. G., \& Hjorth, J. 2000, ApJ, 544, L35

Kochanek, C. S. 1996, ApJ, 473, 595

Lucy, L. B. 1974, AJ, 79, 745

Mathur, S., Elvis, M., \& Singh, K. P. 1995, ApJ, 455, L9

Mohr, J. J., Mathiesen, B., \& Evrard, A. E. 1999, ApJ, 517, 627

Press, W. H., et al. 1995, Numerical Recipes in C. (Cambridge: Cambridge University Press)

Raymond, J. C., \& Smith, B. W. 1977, ApJS, 35, 419

Reimers, D., Bade, N., Schartel, N., et al., 1995, A\&A, 296, L49

Refsdal, S. 1964, MNRAS, 128, 307

Richardson, W. H. 1972, J. Opt. Soc. Am., 62, 55

Roettiger, K., Stone, J. M., \& Mushotzky, R. F. 1998, ApJ, 493, 62

Weisskopf, M. C., O’Dell, S. L. \& van Speybroeck, L. P. 1996, Proc. SPIE, 2805, 2

Witt, H. J., Mao, S., \& Schechter, P. L. 1995, ApJ, 443, 18 
Wise, M. W., Huenemoerder, \& D. P., Davis, J. E. 2000, ADASS VI, A.S.P. Conf. Series, Vol. 125, 1997, ed. Gareth Hunt \& H. E. Payne, p. 477 
Fig. 1.- Panel a: Chandra X-ray image of the quadruply lensed quasar RX J0911.4+0551, resampled at one-fifth of an ACIS pixel (1 ACIS pixel =0".492) and smoothed with a Gaussian of $\sigma=0^{\prime \prime}$. . Panel b: Deconvolution of the Chandra X-ray image using the Richardson-Lucy algorithm. The deconvolved image has been smoothed with a Gaussian of $\sigma=0^{\prime \prime} 2$. The four components of the system are labeled according to the nomenclature of Burud et al. (1998). North is up and East is left; the width of each panel is $\sim 5.5^{\prime \prime}$. See inset of Figure 3 to compare the image configuration with an HST/NICMOS observation of the system.

Fig. 2.- Top panel: Chandra spectral data of the combined quasar images of RX J0911.4+0551 with a best-fit power law (including Galactic and intrinsic absorption) to the hard (1.5-7.0 keV) energy band. Bottom panel: Deviation (in units of one standard deviation) of the observed spectral counts to the hard energy band fit.

Fig. 3.- Solid lines: X-ray contours (logarithmically spaced) of an adaptively smoothed Chandra image of the RX J0911.4+0551 field (excluding emission from RX J0911.4+0551 itself). The background image is a composite optical image taken with the NOT and ESO/NTT (Burud et al. 1998). X-ray contours have been aligned with the optical exposure according to the Chandra aspect solution. Dashed line: Elliptical aperture used in $\S 3$ to estimate the cluster luminosity. Inset: Closeup of RX J0911.4+0551 taken from archival HST/NICMOS imaging of E. Falco (PI). The 4 quasar images and lensing galaxy are labeled. 
Table 1. Optical and X-ray Offsets of RX J0911.4+0551 Components

\begin{tabular}{ccccc}
\hline \hline Telescope & $\mathrm{B}$ & $\mathrm{A} 1$ & $\mathrm{~A} 2$ & $\mathrm{~A} 3$ \\
& $\Delta \alpha\left(^{\prime \prime}\right), \Delta \delta\left(^{\prime \prime}\right)$ & $\Delta \alpha\left(^{\prime \prime}\right), \Delta \delta\left(^{\prime \prime}\right)$ & $\Delta \alpha\left(^{\prime \prime}\right), \Delta \delta\left(^{\prime \prime}\right)$ & $\Delta \alpha\left(^{\prime \prime}\right), \Delta \delta\left(^{\prime \prime}\right)$ \\
\hline NOT & $0.00,0.00$ & $2.935 \pm 0.004,-0.785 \pm 0.009$ & $3.194 \pm 0.007,-0.383 \pm 0.007$ & $2.922 \pm 0.008,0.161 \pm 0.009$ \\
Chandra & $0.00,0.00$ & $2.88 \pm 0.20,-0.88 \pm 0.20$ & $3.32 \pm 0.20,-0.41 \pm 0.20$ & $2.88 \pm 0.20,0.17 \pm 0.20$ \\
\hline
\end{tabular}

Note. - Optical positions and corresponding $1 \sigma$ error bars are from observations obtained with the Nordic Optical Telescope (NOT; Burud et al. 1998). Relative offsets are in the sense of component A minus component B. 
Table 2. Contemporaneous Optical and X-ray Flux Ratios of RX J0911.4+0551 Components

\begin{tabular}{cccc}
\hline \hline Waveband & $(\mathrm{A} 1+\mathrm{A} 2) / \mathrm{B}$ & $(\mathrm{A} 1+\mathrm{A} 2+\mathrm{A} 3) / \mathrm{B}$ & $(\mathrm{A} 3) / \mathrm{B}$ \\
\hline I band & $5.67 \pm 0.14$ & $6.95 \pm 0.16$ & $1.28 \pm 0.07$ \\
$0.3-7.0 \mathrm{keV}$ & $4.68_{-0.20}^{+0.30}$ & $4.87_{-0.12}^{+0.23}$ & $0.19_{-0.17}^{+0.24}$ \\
\hline
\end{tabular}

Note. - I-band ratios and corresponding $1 \sigma$ error bars are from an ongoing monitoring campaign of RX J0911.4+0551 with the Nordic Optical Telescope (Hjorth et al. 2001). Error bars for the X-ray ratios represent $90 \%$ confidence intervals. 


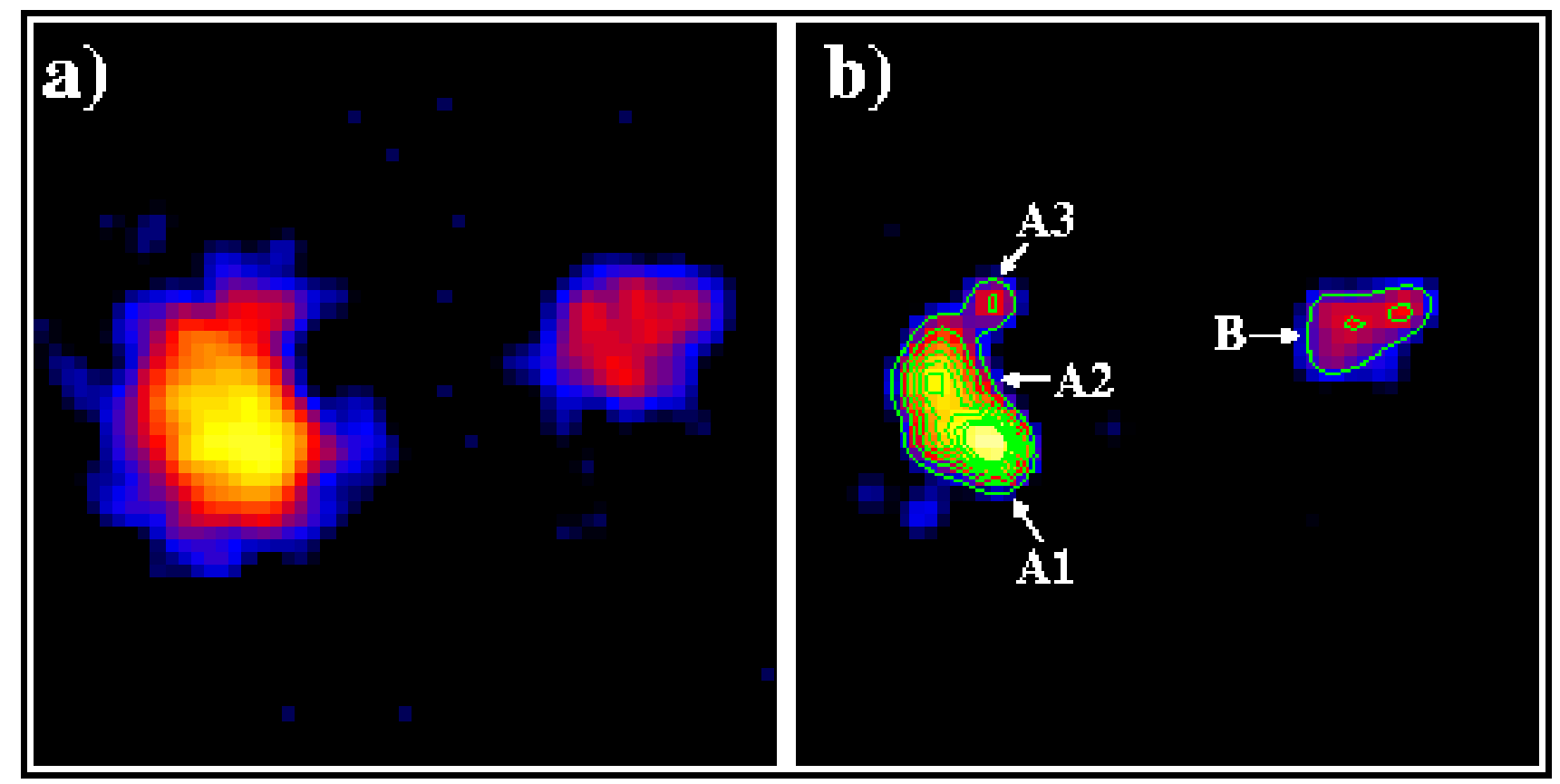




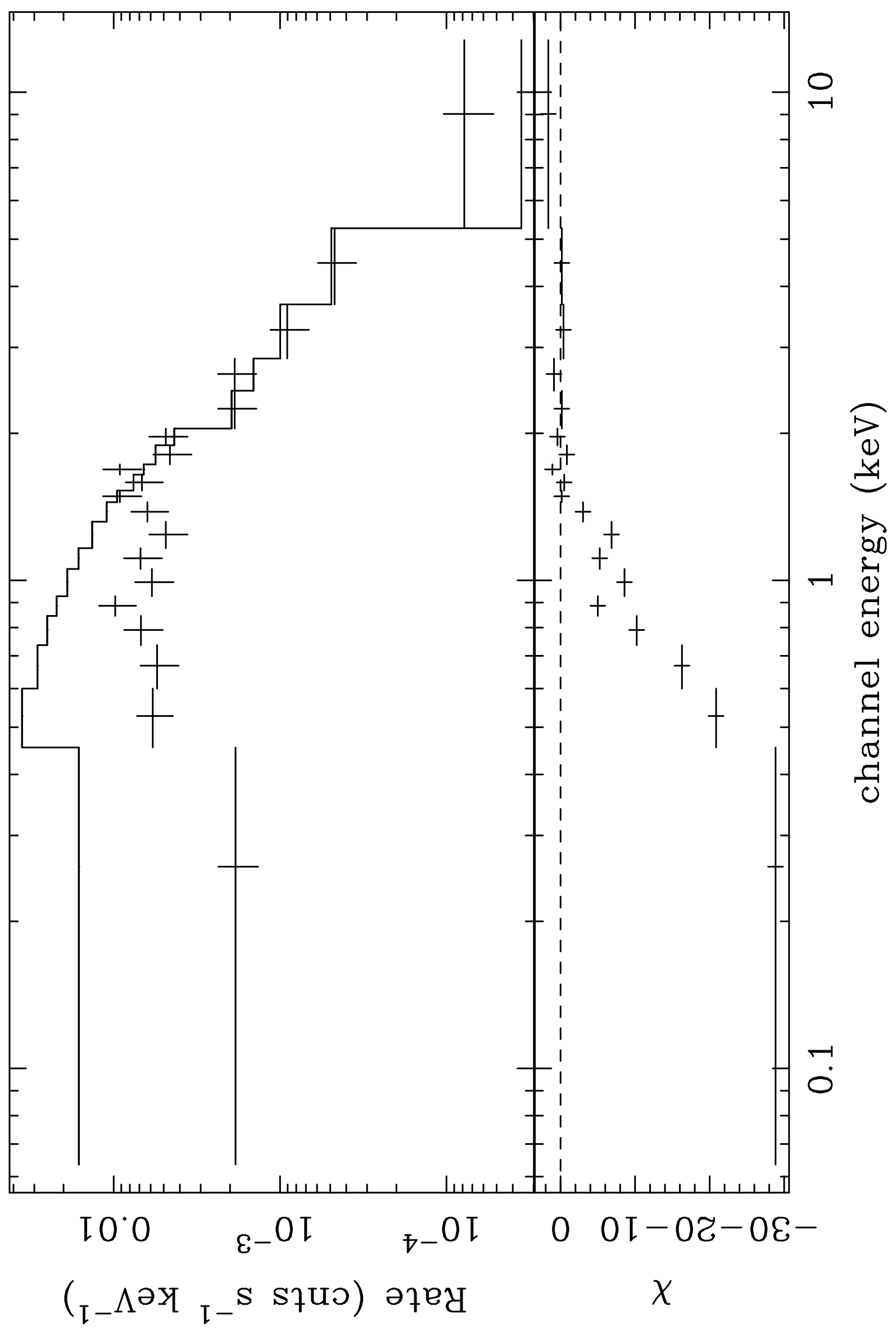




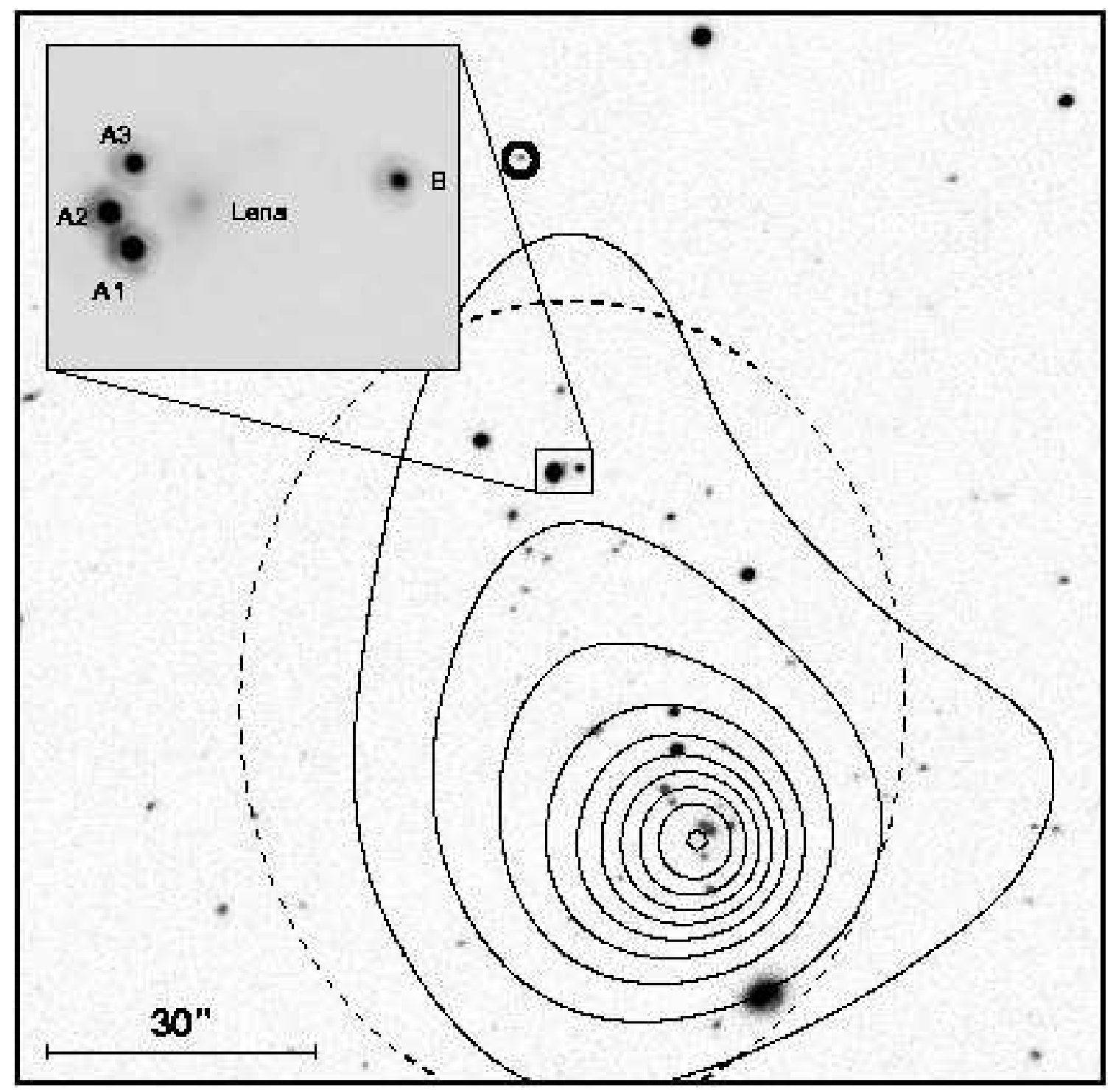

